\title{
Efectos de la acupuntura sobre la secreción lagrimal (acupuntos BL-2, TH-23 y Tai-Yang) y la presión intraocular (acupuntos LI-4, LIV-3 y GB-37) en perros de raza Beagle
}

\author{
Effects of acupuncture on tear secretion (BL-2, TH-23 and Tai-Yang acupoints) and intraocular \\ pressure (LI-4, LIV-3 and GB-37 acupoints) in beagle dogs \\ JC Silva ${ }^{\text {a*, MC Tovar }}{ }^{\mathrm{a}}$, FG Cano ${ }^{\mathrm{b}}, \mathrm{J}$ Zilberschtein ${ }^{\mathrm{a}}$ \\ aDepartamento de Medicina y Cirugía Animal, Facultad de Veterinaria,Universidad de Murcia, Murcia, España. \\ bDepartamento de Anatomía y Anatomía Patológica Comparadas, Facultad de Veterinaria, Universidad de Murcia, Murcia, España.
}

\section{SUMMARY}

\begin{abstract}
The aim of the study is to evaluate the effect of the acupuncture technique on tear secretion (TS) and intraocular pressure (IOP). Ten healthy beagle dogs were enrolled in the study. The acupoints BL -2, TH- 23 and Tai-yang (orbital region) were stimulated within 30 minutes to assess their influence on the TS. On the other hand, modifications of the IOP were evaluated after stimulating the acupoints LI -4 (upper limb), and LIV-3 and GB-37 (hind limb). TS and IOP were assessed by Schirmer test and applanation tonometer, respectively. All data was obtained at the same time intervals: $\mathrm{T} 0=\mathrm{control}$ values, T5 (5 min), T15 (15 min), T30 (30 $\mathrm{min})$ and T60 $(60 \mathrm{~min})$ from the withdrawal of the needles. Stimulation of the TS acupoints showed the following results: T0 $18.95 \pm 3.60 \mathrm{~mm} / \mathrm{min}$; T5 $24.50 \pm 3.79 \mathrm{~mm} / \mathrm{min}$; T15 $25.70 \pm 3.91 \mathrm{~mm} / \mathrm{min} ; \mathrm{T} 3025.90 \pm 4.75 \mathrm{~mm} / \mathrm{min} ; \mathrm{T} 6021.90 \pm 5.09 \mathrm{~mm} / \mathrm{min}$. Stimulation of the IOP acupoints showed the following results: T0 $22.00 \pm 2.06 \mathrm{mmHg}$; T5 $15.90 \pm 1.57 \mathrm{mmHg}$; T15 $13.70 \pm 1.65 \mathrm{mmHg}$; T30 12.65 $\pm 1.70 \mathrm{mmHg}$; T60 $13.95 \pm 1.83 \mathrm{mmHg}$. The results obtained show that acupuncture technique in dogs might be useful in the treatment the pathologies involving tear hyposecretion and/or increased intraocular pressure.
\end{abstract}

Key words: acupuncture, acupoints, intraocular pressure, tear secretion, dog.

\section{RESUMEN}

El objetivo del estudio fue evaluar el efecto de la técnica de acupuntura sobre la secreción lagrimal (SL) y la presión intraocular (PIO). Diez perros Beagle sanos se usaron en el estudio. Para valorar la SL se estimularon los acupuntos BL-2, TH-23 y Tai-yang (región orbitaria), mientras que para analizarlos valores de la PIO se estimularon los acupuntos LI-4 (miembro torácico) y LIV-3, GB-37 (miembro pelviano), durante 30 minutos. Se utilizó el test lagrimal de Schirmer para valorar la SL, y el tonómetro de aplanación para la PIO. Los valores resultantes fueron obtenidos en diferentes tiempos de lectura: $\mathrm{T} 0=$ valores control (sin estimulación), T5, T15, T30 y T60, referidos a 5, 15, 30 y 60 minutos transcurridos desde la retirada de las agujas. Los resultados obtenidos para la SL: T0 de 18,95 $\pm 3,60 \mathrm{~mm} / \mathrm{min}$; T5de 24,50 $\pm 3,79 \mathrm{~mm} / \mathrm{min}$; T15de 25,70 $\pm 3,91 \mathrm{~mm} / \mathrm{min} ; \mathrm{T} 30 \mathrm{de} 25,90 \pm 4,75 \mathrm{~mm} /$ $\mathrm{min}$; T60 de 21,90 \pm 5,09 mm/min. Los resultados obtenidos respecto de la PIO: T0 de 22,00 $\pm 2,06 \mathrm{mmHg}$; T5 de 15,90 $\pm 1,57 \mathrm{mmHg}$; T15de 13,70 $\pm 1,65 \mathrm{mmHg}$; T30 de 12,65 \pm 1,70 mmHg; T60 de 13,95 \pm 1,83 mmHg. Los mejores resultados en ambas pruebas se obtuvieron a los 30 minutos de la retirada de las agujas. Los resultados muestran que las técnicas de acupuntura en perros podrían ser útiles en el tratamiento de procesos que cursen con hiposecreción lagrimal o aumento de la presión intraocular.

Palabras clave: acupuntura, acupuntos, presión intraocular, secreción lagrimal.

\section{INTRODUCCIÓN}

El diagnóstico clínico y el tratamiento de una afección ocular representan un reto para muchos de los veterinarios, generalmente poco familiarizados con la patología oftálmica. Sin embargo, algunas de estas patologías se presentan frecuentemente en la clínica diaria. Un lugar de primer orden ocupan las enfermedades relacionadas con la disminución de la secreción lagrimal (SL), muy frecuente en la raza canina, y el aumento de la presión intraocular

Aceptado: 07.05.2015.

jocasvet@hotmail.com
(PIO); siendo esta última, al igual que ocurre en la especie humana, una de las principales causas de ceguera.

La lágrima tiene una gran importancia en la estabilidad y viabilidad del epitelio conjuntival y corneal debido a la dependencia que existe entre las distintas estructuras que integran la superficie ocular (Tseng y col 2006). La córnea obtiene sus principales nutrientes (glucosa, electrolitos, etc.) desde el humor acuoso, pero los factores de crecimiento, vitaminas y neuropéptidos responsables de la proliferación, migración y diferenciación de las células del epitelio conjuntival y corneal provienen de la lágrima y también son vertidos a ella los desechos resultantes de su metabolismo (Barton y col 1998, Wilson y col 1999, López y col 2007). Además, las lágrimas tienen propiedades antimicrobianas, nutritivas, mecánicas y ópticas (Geerling y col 2004). En casos de sequedad ocular la toxicidad sobre las células 
epiteliales está aumentada, siendo común la presencia de trastornos epiteliales (Burstein 1985, Gobbels y col 1992, López y col 2007).

En condiciones anormales puede haber una disminución de su secreción, lo que conocemos como hiposecreción lagrimal, y surge por una disfunción de dichas glándulas. La inflamación corneal crónica que se produce por esta causa puede conducir a ulceración, cicatrización, pigmentación o neovascularización que eventualmente puede causar pérdida de la visión, produciendo una patología ocular conocida como queratoconjuntivitis seca (Grahn y Storey 2004).

Entre los contenidos del ojo está el humor acuoso, un fluido incoloro de baja viscosidad que proviene de un filtrado del plasma y baña las cámaras anterior y posterior, cuyo volumen determina la PIO. El aumento de esta presión es una de las principales causas de ceguera en el perro (Maggs y col 2009). Sus primeras manifestaciones son: degeneración y muerte de las células ganglionares de la retina (CGR), degeneración de axones del nervio óptico y por tanto disminución de la capa de fibras nerviosas de la retina (CFNR), aumento de la excavación papilar, disminución del anillo neurorretiniano (ANR) y desestabilización de la zona de origen (cabeza) del nervio óptico ${ }^{1}$. Es lo que se conoce, tanto en medicina humana como en veterinaria, como glaucoma.

El tratamiento de la hiposecreción lagrimal y del aumento de la PIO consiste en la administración de diversos fármacos o la realización de cirugías que resuelvan, de uno u otro modo, estas situaciones. En determinados casos, la medicina convencional no consigue un resultado satisfactorio en el tratamiento de estas alteraciones oculares, y se convierten en procesos crónicos con escasas o nulas posibilidades de éxito. Por esta razón se plantea este estudio, para la aplicación de otras técnicas alternativas menos frecuentes, pero no por ello menos eficaces, empleadas en otros ámbitos de la medicina veterinaria y que podrían ser muy útiles en oftalmología.

La acupuntura es una técnica terapéutica desarrollada por los médicos chinos hace más de cinco mil años. Esta técnica consiste en la inserción de agujas metálicas esterilizadas en puntos con una localización definida (acupuntos), de forma manual o con electroestimulación (electroacupuntura), produciendo una acción específica que genera, entre otros, efectos analgésicos, antiinflamatorios e inmunoestimulantes. La selección de los puntos de acupuntura se define por un diagnóstico enfocado en los principios de las bases de la medicina tradicional china (MTCh) y es individual para cada paciente. El uso en las pequeñas especies es relativamente nuevo, adaptándose el

1 Molleda JM, EM Martín-Suárez. 2009. Claves para el diagnóstico precoz del glaucoma. REDVET. Revista electrónica de Veterinaria. ISSN: 1695-7504 Vol. 10, $\mathrm{N}^{\circ} 3$. Disponible en http://www.veterinaria. org/revistas/redvet/n030309.html. sistema de meridianos y de acupuntos de los humanos al perro y al gato con muy buenos resultados (Rodekohr 2002).

Respecto del empleo de la técnica en oftalmología, los datos científicos en los animales son limitados. Así, la mayor parte de ellos han sido obtenidos a partir de ensayos clínicos realizados en humanos (Naruse y col 2000) y a partir de investigaciones hechas en animales de laboratorio (Chu y Potter 2002). En la actualidad, es una técnica ampliamente aceptada para el tratamiento de una gran variedad de condiciones (Ernest 2006). Según algunos autores, muchos de los trastornos oculares pueden ser tratados con acupuntura, por ejemplo, conjuntivitis alérgicas, úlceras de córnea, glaucomas, cataratas, ojo seco (Bensky y O’Connor 1981, Dabov y col 1985, Ross 1989, Cariello y col 2006) y síndrome de Horner (Cho y Kim 2008), entre otros.

En los humanos existen más de 300 acupuntos que se comunican entre sí y actúan de manera individual o en conjunto en numerosas patologías. En la literatura disponible se encuentran estudios realizados para el tratamiento de "sequedad ocular" utilizando una gran variedad de acupuntos, salvo para dos acupuntos específicos, el BL-2 y el Tai-yang que son puntos periorbitarios utilizados en la mayor parte de las investigaciones. A estos puntos se ha de añadir un tercero, el TH-23, ya que esta triada ha sido utilizada anteriormente en conejos con resultados significativos (Gong y Sun 2007).

Hasta la fecha no hay ninguna publicación sobre el uso de la acupuntura en la regulación de la secreción lagrimal en perros; y respecto de la PIO tan solo existen dos para la especie canina (Kim y col 2005, Kim y col 2007). El objetivo de este estudio experimental es valorar el efecto de la estimulación de los acupuntos perioculares BL-2, TH-23 y Tai-yang sobre la secreción lagrimal y de la estimulación de los acupuntos del miembro torácico LI-4 y miembro pelviano, LIV-3 y GB-37 sobre la PIO en perros sanos.

\section{MATERIAL Y MÉTODOS}

\section{ANIMALES}

Se utilizaron 10 perros de la raza Beagle, cinco machos y cinco hembras, con edades comprendidas entre dos y seis años, pertenecientes al Servicio de Animales de Laboratorio de la Universidad de Murcia, España, siguiendo en todo momento las directrices de la Unión Europea respecto de la protección de animales de experimentación.

Se seleccionaron animales tranquilos, con historial clínico libre de enfermedades oculares y que no presentaran signos de enfermedades sistémicas en el momento del estudio. Para ello, 48 horas antes del inicio de la prueba experimental se realizó a cada perro una reseña y un examen oftalmológico basal que consistió en la identificación del animal (edad, sexo, raza y peso), valoración de la respuesta de amenaza, reflejos palpebrales y fotomotores directos 

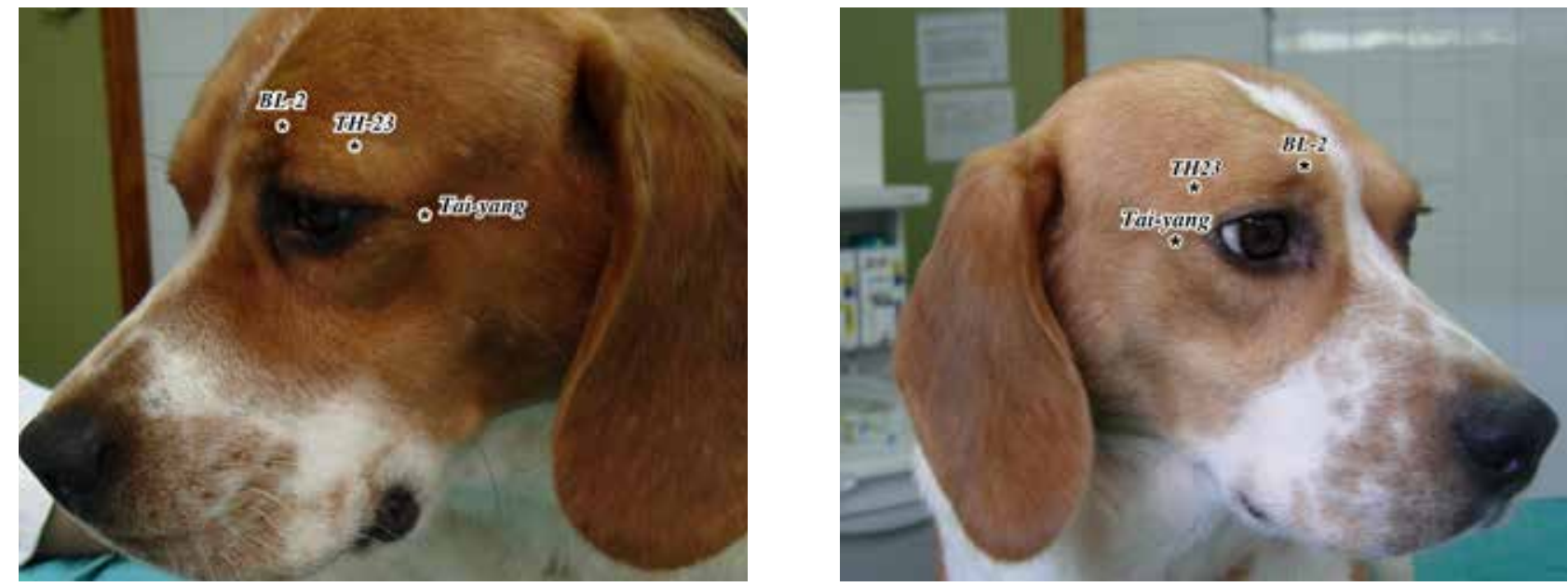

Figura 1 y 2. Vista lateral izquierda y lateral derecha de los acupuntos. BL-2, TH-23 y Tai-yang.

Lateral view left and right side of the acupoints. BL-2, TH-23 and Tai-yang.

y consensuados, examen con lámpara de hendidura del segmento anterior y oftalmoscopia indirecta y directa para examinar el segmento posterior.

\section{MATERIALES}

Ohmiómetro (WQ-IOC2® Multiple Electronic Acupunctoscope, China) para la medición de la resistencia en la superficie de la piel y localizar exactamente los puntos de acupuntura. Agujas chinas de acupuntura desechables con mango de cobre y cabeza redonda sin guía 0,18 x 7 mm (Acimut CE0197®) para los acupuntos perioculares, BL-2, TH-23 y Tai-yang. Agujas chinas de acupuntura desechables con mango de cobre y cabeza redonda sin guía $0,22 \times 13 \mathrm{~mm}(\mathrm{C} \& \mathrm{G}$, Acupuncture Needle CT1/CT5®) para los acupuntos LI-4, LIV-3 y GB-37. Test Lagrimal de Schirmer (Schirmer Tear Test ${ }^{\circledR}$, Schering-Plough Animal Health, Union, New Jersey, USA) para medir la secreción lagrimal. Tonómetro de aplanación (Tono-Pen ${ }^{\circledR X L}$, mentor Medical Systerms, Leiden, Netherlands) para medir la presión intraocular. Colirio anestésico (Colicursí anestésico doble ${ }^{\circledR}$, Alcon Cusí, Barcelona, España) para insensibilizar la córnea previamente a la aplicación del tonómetro.

\section{DISEÑO EXPERIMENTAL}

Todos los perros estudiados fueron integrados, en una primera fase, en el llamado grupo control T0, ya que antes de la aplicación de las agujas de acupuntura se tomaron mediciones de la SL y de la PIO, cuyos valores fueron considerados como de referencia. En una segunda fase, estos mismos animales se destinaron al estudio experimental con objeto de medir la SL y la PIO después de la aplicación de las agujas.

La localización de todos los acupuntos se determinó mediante la utilización de un ohmiómetro según descripción anatómica (Xie y Preast 2007).
En los acupuntos perioculares BL-2, TH-23 y Tai-yang (figuras 1 y 2), destinados a medir la influencia de la acupuntura sobre la SL, se hizo la inserción de las agujas. Asimismo, para la prueba de la PIO se hizo también la inserción de agujas en los acupuntos LI-4 (figura 3) en los miembros torácicos y LIV-3 (figuras 4 y 5) y GB-37 (figura 6) en los miembros pelvianos. La localización e inserción de las agujas en estos acupuntos se hizo siempre de forma bilateral.

Se obedeció a un mismo patrón de cinco tiempos. Los tiempos se marcaron desde que se retiraban las agujas de

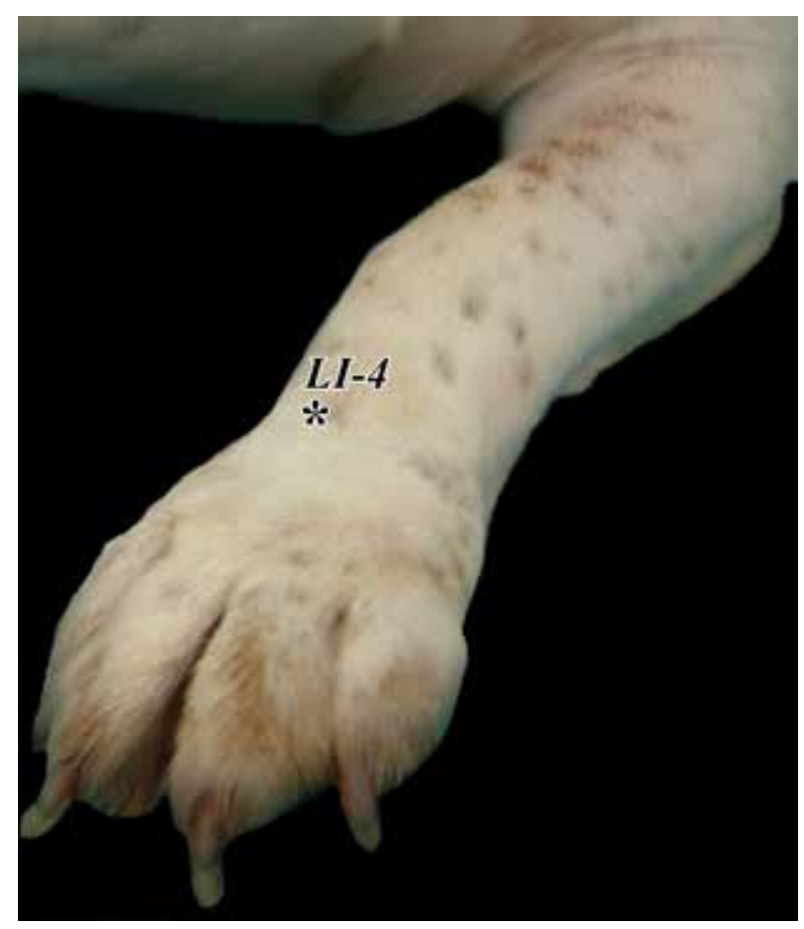

Figura 3. Acupunto LI-4. Acupoint LI-4. 

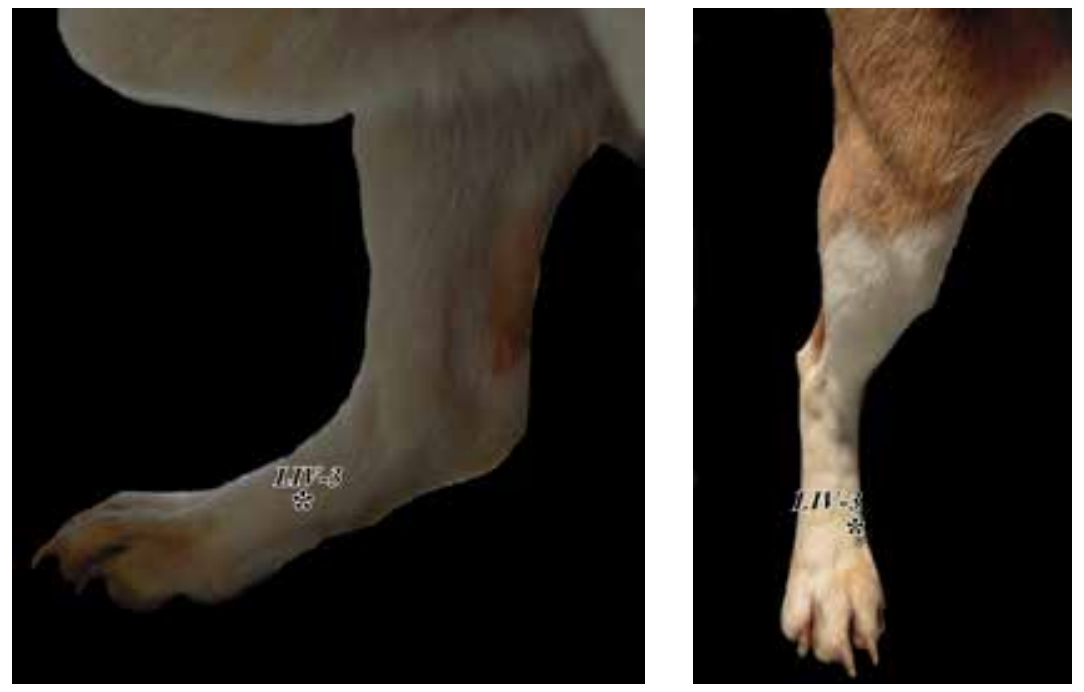

Figura 4 y 5. Acupunto LIV-3: Vista craneal y medial. Acupoint LIV-3. Cranial and medial Vista.
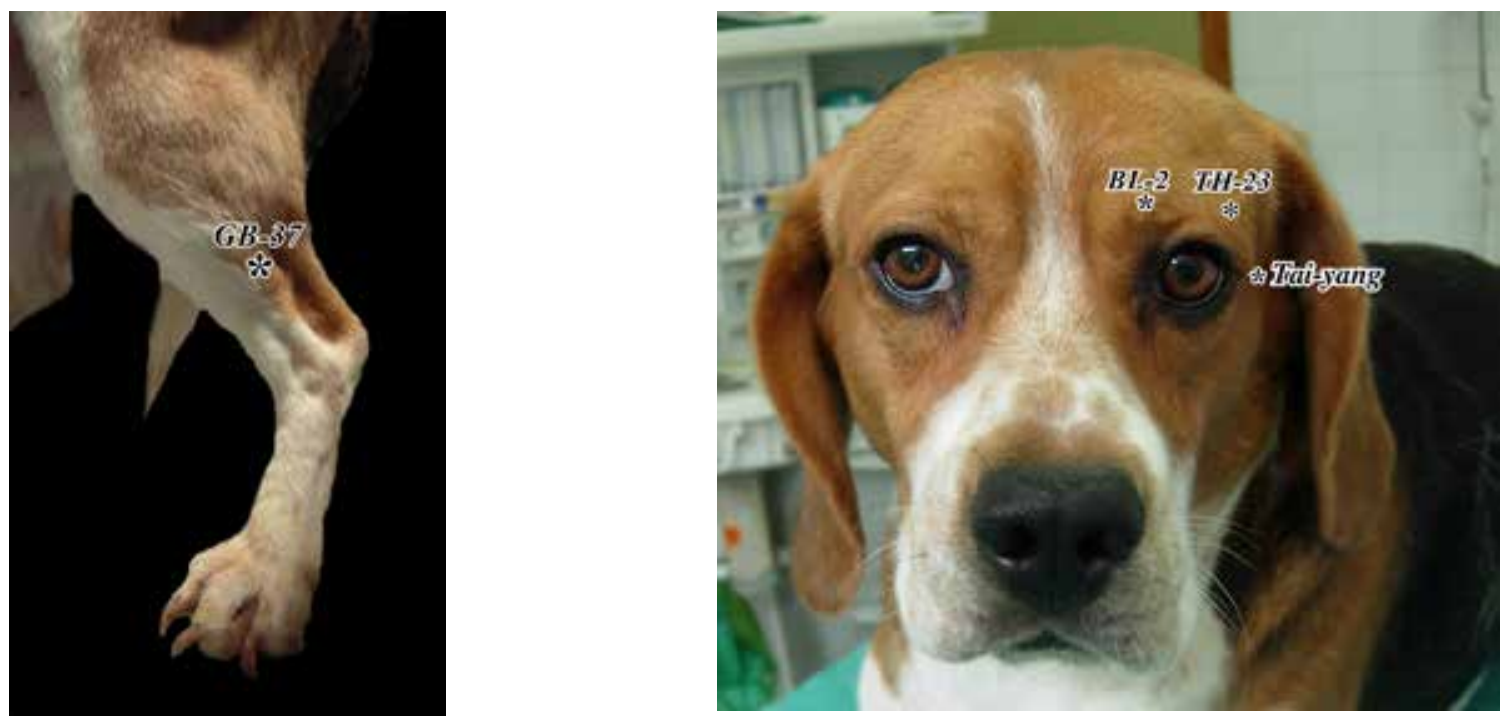

Figura 6. Acupuntos GB-37. Acupoints GB-37.

Figura 7. inserción de las agujas en los acupuntos BL-2, TH-23 y Tai-yang.

Insertion needles in the BL-2, TH-23 and Tai-yang acupoints.

acupuntura, hasta que se realizaba la lectura de la medición de la SL o de la PIO. El tiempo 0 (T0) se consideró a la toma de mediciones previamente a la aplicación de las agujas, actuando este como valor control. Las medidas de los valores obtenidos se realizaron en intervalos de 5 (T5), 15 (T15), 30 (T30) y 60 (T60) minutos después de la retirada de las agujas. El tiempo destinado a la estimulación de los acupuntos fue de 30 minutos.

Estas medidas a distintos tiempos se tomaron en cada uno de los ojos de cada animal en los dos experimentos diseñados, dejando un período de una semana entre ellos, de forma que no interfirieran entre sí las dos técnicas.

\section{EVALUACIÓN DE LA SL DESPUÉS DE ESTIMULAR LOS} ACUPUNTOS BL-2, TH- 23 Y TAI-YANG

La prueba de la secreción lagrimal se realizó tras un periodo de adaptación de los animales, cuando se encontraban en un estado de relajación casi completo, no habiendo manipulación previa y no utilizando en ningún momento anestésicos o sedación. La producción de secreción lagrimal fue evaluada en los 10 animales (en los dos ojos) utilizando el test lagrimal de Schirmer en los cinco tiempos distintos: T0, T5, T15, T30 y $\mathrm{T} 60$. 
Los acupuntos utilizados fueron los siguientes (figura 7):

$B L-2$ : se encuentra en la cresta supraorbitaria, por debajo del extremo medial en la escotadura supraorbitaria.

$T H$-23: se encuentra en una depresión palpable en el borde de la órbita, que se extiende al canto lateral de la órbita.

Tai-yang: ubicado en una depresión palpable en los lados de la cabeza a $1 \mathrm{~cm}$ lateral canto temporal del ojo.

En todos los casos la aguja se inserta oblicua hacia abajo a una profundidad de 0,3 a $0,5 \mathrm{~cm}$. El tiempo de estimulación es de 30 minutos.

\section{EVALUACIÓN DE LA PIO DESPUÉS DE ESTIMULAR LOS ACUPUNTOS LI-4, LIV-3 Y GB-37}

Para la prueba de medición de la PIO, el procedimiento de adaptación fue similar, dejando pasar una semana de reposo a los animales desde que se realizó el estudio para la SL. La medición de la PIO se realizó mediante un tonómetro de aplanación en los cinco tiempos descritos anteriormente: T0, T5, T15, T30 y T60. Todas las mediciones fueron tomadas un minuto después de la aplicación del colirio anestésico tópico, intentando no provocar estrés al animal y teniendo la precaución de no presionar sobre el cuello ni sobre estructuras blandas perioculares.

Los acupuntos utilizados fueron los siguientes (figura 8):

LI-4: situado en el miembro torácico, entre el segundo y tercer huesos metacarpianos a nivel de la zona media de este último hueso.

LIV-3: ubicado en el miembro pelviano, concretamente en la región del metatarso, proximal a la unión entre el segundo y tercer huesos metatarsianos, por su cara dorsal.

GB-37: localizado en el miembro pelviano, en la cara lateral de la pierna a nivel del tercio medio desde el extremo del maléolo lateral de la tibia al borde craneal de la cabeza del peroné.

En todos los casos la aguja se inserta oblicua hacia abajo a una profundidad de 0,5 a $0,8 \mathrm{~cm}$. Tiempo de estimulación 30 minutos.

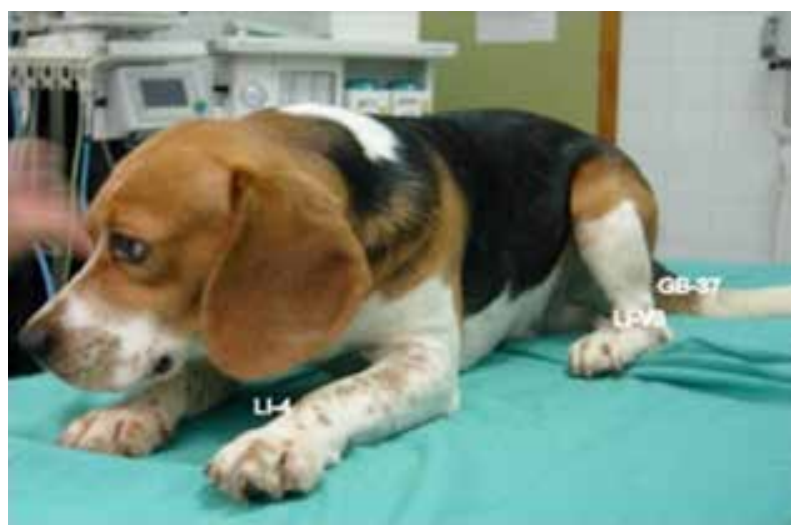

Figura 8. Inserción de las agujas en los acupuntos LI-4, LIV-3 y GB-37.

Insertion needles in to LI-4, LIV-3 and GB-37 acupoints.

\section{ANÁLISIS ESTADÍSTICO}

Para el análisis estadístico de los datos se empleó el paquete informático SPSS 15.0.1, para Windows y la hoja de cálculo de Microsoft Excel 2002.

Se realizó un análisis descriptivo, obteniendo la media, desviación típica y valores máximo y mínimo para los datos de la SL y de la PIO, en cada uno de los animales. Se detectó normalidad y homocedasticidad para todas las variables.

Los valores de las medias antes y después de la estimulación, en cada una de las experiencias realizadas fueron comparados mediante la prueba " $t$ " de student para muestras pareadas con un nivel de significación de 0,05 $(\mathrm{P}<0,05)$. También se usó esta prueba para evaluar las variaciones dentro de las distintas experiencias. Aplicamos el método de análisis de varianza (ANOVA) de medidas repetidas para analizar el efecto del tiempo de estimulación en un mismo individuo, comparando los promedios entre ojo derecho y ojo izquierdo y analizando la significación estadística de cada uno de los valores obtenidos. Tras este análisis, con las variaciones que mostraron diferencias estadísticamente significativas, se realizaron comparaciones por pares mediante el test de Bonferroni para precisar el origen de dichas diferencias.

\section{RESULTADOS}

\section{EVALUACIÓN DE LA SL DESPUÉS DE ESTIMULAR LOS} ACUPUNTOS BL-2, TH- 23 Y TAI-YANG

El aumento de la SL fue obtenido en 20 ojos de los 10 perros estudiados, su valor medio y desviación estándar (DE) fue para el T0 de 18,95 $\pm 3,60 \mathrm{~mm} / \mathrm{min}$; T5 de $24,50 \pm 3,79 \mathrm{~mm} / \mathrm{min}$; T15 de $25,70 \pm 3,91 \mathrm{~mm} / \mathrm{min}$; T30 de $25,90 \pm 4,75 \mathrm{~mm} / \mathrm{min}$; T60 de 21,90 $\pm 5,09 \mathrm{~mm} / \mathrm{min}$ respectivamente, existiendo una diferencia significativa en relación con el tiempo control $(\mathrm{T} 0)(\mathrm{P}<0,05)$ (cuadro 1).

Según estos resultados, la estimulación de los acupuntos estudiados demuestra que los valores de la SL varían en función del tiempo empleado. Así, observamos que en el T5 aumentó un promedio de $5,5 \mathrm{~mm} / \mathrm{min}$, es decir, un $29,2 \%$ superior respecto del T0. En el T15 se pudo observar también un incremento de $6,7 \mathrm{~mm} / \mathrm{min}$, lo que representa un $35,6 \%$ más respecto de T0. En el T30 se siguió observando un incremento de estos valores siendo de $6,9 \mathrm{~mm} / \mathrm{min}$, o sea 36,6\% más en relación con el tiempo inicial. Durante el T60 comienza una disminución de la SL respecto del tiempo anterior (T30), siendo su valor de $4 \mathrm{~mm} / \mathrm{min}$, es decir, $15,4 \%$ menos. Sin embargo en relación con el T0, la diferencia fue de $2,95 \mathrm{~mm} / \mathrm{min}$, un $15,6 \%$ superior.

Como se puede apreciar la técnica de acupuntura produce un aumento de la secreción lagrimal en los 20 ojos $(\mathrm{N}=20)$ de los 10 perros estudiados, existiendo una diferencia significativa en relación con el tiempo control $\mathrm{T} 0(\mathrm{P}=<0,05)$. Las comparaciones por pares (de tiempos) 
Cuadro 1. Valores medios de secreción lagrimal en los diferentes tiempos analizados.

Mean values of tear secretion in the different time points analyzed.

\begin{tabular}{lccccc}
\hline & T0 & T5 & T15 & T30 & T60 \\
\hline $\begin{array}{l}\text { Media } \\
\text { N=20) }\end{array}$ & 18,95 & 24,55 & 25,70 & 25,90 & 21,90 \\
$\begin{array}{l}\text { DE } \\
\text { Aumento de la SL en compa- }\end{array}$ & 3,60 & 3,79 & 3,91 & 4,75 & 5,09 \\
ración al T0 & - & $5,6 \mathrm{~mm} / \mathrm{min}$ & $6,7 \mathrm{~mm} / \mathrm{min}$ & $6,9 \mathrm{~mm} / \mathrm{min}$ & $2,9 \mathrm{~mm} / \mathrm{min}$ \\
\hline
\end{tabular}

$\mathrm{T}=$ tiempo en minutos. $\mathrm{N}=$ número de ojos. $\mathrm{DE}=$ desviación estándar. SL= secreción lagrimal

$\mathrm{T}=$ time in minutes. $\mathrm{N}=$ number of eyes. $\mathrm{DE}=$ deviation estándar. $\mathrm{SL}=$ tear secretion.

Cuadro 2. Comparaciones por pares, solo resultados significativos. Basadas en las medias marginales estimadas. Summary of pairwise comparisons, only significant results, based on estimated marginal means.

\begin{tabular}{ccc}
\hline Tiempo control & Tiempo de medición & Diferencia entre medias $(\mathrm{C}-\mathrm{M})$ \\
\hline 0 & 5 & $-5,600\left(^{*}\right)$ \\
0 & 15 & $-6,750\left(^{*}\right)$ \\
0 & 30 & $-6,950\left(^{*}\right)$ \\
0 & 60 & $-2,950$ \\
\hline
\end{tabular}

(C-M) diferencia entre tiempo control y tiempo de medición. *La diferencia de las medias es significativa al nivel 0,05.

(C-M) difference between control and measurement time period. *The mean difference is significant at the 0,05 level.

mostraron una diferencia significativa entre los tiempos 0 , respecto de los tiempos 5,15 y 30 , no existiendo una diferencia significativa con el tiempo 60 (cuadro 2).

Por tanto, los valores extremos del SL en este grupo de estudio estuvieron entre un valor mínimo de $18,95 \mathrm{~mm} /$ min y un máximo de $25,90 \mathrm{~mm} / \mathrm{min}$. Existió un incremento en todos los tiempos después de la estimulación, siendo el mejor resultado el obtenido en el tiempo T30.

\section{EVALUACIÓN DE LA PIO DESPUÉS DE ESTIMULAR LOS ACUPUNTOS LI-4, LIV-3 Y GB-37}

La disminución de la PIO fue obtenida en 20 ojos de los 10 perros estudiados, su valor medio y desviación estándar (DE) fue para el T0 de 22,00 $\pm 2,06 \mathrm{mmHg}$; T5 de 15,90 \pm 1,57 mmHg; T15 de 13,70 \pm 1,65 mmHg; T30 de 12,65 \pm 1,70 mmHg; T60 de 13,95 \pm 1,83 mmHg respectivamente (cuadro 3 ).

$\mathrm{Si}$ analizamos los tiempos empleados en el experimento, los valores de la PIO con esta técnica demuestran que en el T5 disminuyó un promedio de $6,2 \mathrm{mmHg}$, es decir, $27,7 \%$ respecto del T0. Hubo también una reducción en el T15 de 8,3 mmHg, 37,7\% respecto del T0. En el T30 se observó una disminución en los valores de 9,3 $\mathrm{mmHg}$, o sea, $42,5 \%$ respecto al tiempo inicial. Sin embargo, durante el T60 se observó un aumento de la PIO respecto del T30 de $1,3 \mathrm{mmHg}$, es decir, $10,3 \%$, si bien en relación con el T0, la diferencia fue de $8,05 \mathrm{mmHg}$ menos, el 36,6\%.

Como se puede observar la técnica de acupuntura produce una disminución de la PIO en los 20 ojos $(\mathrm{N}=20)$ de los 10 perros estudiados, existiendo una diferencia significativa en relación con el tiempo control T0 $(\mathrm{P}=<0,05)$. Las comparaciones por pares (de tiempos) mostró una diferencia significativa entre los tiempos 0 , respecto de todos los tiempos (cuadro 4).

Por tanto, los valores extremos de PIO tras la aplicación de las agujas de acupuntura oscilaron entre 12,65 mmHg (valor mínimo) y 22,00 mmHg (valor máximo), existiendo una disminución en todos los tiempos después de la estimulación siendo el mejor resultado el obtenido a los 30 minutos (T30).

\section{DISCUSIÓN}

La acupuntura se ha considerado una alternativa a la medicina occidental y son numerosos los autores que han hecho experiencias en ese campo (Bensky y O'Connor 1981, Dabov y col 1985, Ross 1989, Chu y Potter, 2002, Kim y col 2005, Cariello y col 2006, Gong y Sun 2007, Cho y Kim 2008). Sabemos que la acupuntura reacciona a nivel local, en la medula espinal, y a nivel general, en el cerebro (Gronlund y col 2004). Algunas investigaciones confirman que a nivel ocular, estimulaciones nerviosas originan vasodilatación y la liberación de neuropéptidos y neurotransmisores, que actúan como factores endógenos antiinflamatorios e inhibidores del dolor (Müller y col 2003).

En relación con nuestra experiencia con los acupuntos empleados para evaluar su influencia sobre la secreción lagrimal, los acupuntos BL-2, TH-23 y Tai-yang, podemos decir que por tener una localización periocular, 
Cuadro 3. Valores medios de la PIO en los diferentes tiempos analizados.

Mean values of IOP at different times analyzed.

\begin{tabular}{lccccc}
\hline & T0 & T5 & T15 & T30 & T60 \\
\hline Media(N=20) & 22,0 & 16,0 & 14,0 & 12,7 & 14,0 \\
DE & 2,1 & 1,6 & 1,7 & 1,7 & 1,83 \\
$\begin{array}{l}\text { Disminución de la PIO en } \\
\text { comparación al T0 }\end{array}$ & - & $6,2 \mathrm{mmHg}$ & $8,3 \mathrm{mmHg}$ & $9,3 \mathrm{mmHg}$ & $8,1 \mathrm{mmHg}$ \\
\hline
\end{tabular}

$\mathrm{T}=$ tiempo. $\mathrm{N}=$ número de ojos. $\mathrm{DE}=$ desviación estándar. $\mathrm{PIO}=$ presión intraocular.

$\mathrm{T}=$ time. $\mathrm{N}=$ number of eyes. $\mathrm{DE}=$ desviation estándar. $\mathrm{IOP}=$ intraocular pressure.

Cuadro 4. Comparaciones por pares, solo resultados significativos. Basadas en las medias marginales estimadas. Summary of pairwise comparisons, only significant results, based on estimated marginal means.

\begin{tabular}{ccc}
\hline tiempo control & tiempo de medición & Diferencia entre medias (C- M) \\
\hline 0 & 5 & $-8,250(*)$ \\
0 & 15 & $-10,700(*)$ \\
0 & 30 & $-10,950\left(^{*}\right)$ \\
0 & 60 & $-7,700(*)$ \\
\hline
\end{tabular}

(C-M) diferencia entre tiempo control y tiempo de medición.*La diferencia de las medias es significativa al nivel 0,05.

(C-M) difference between control and measurement time period. ${ }^{*}$ The mean difference is significant at the 0.05 level.

son fácilmente identificables. Se encuentran en lugares específicos que involucran los nervios trigémino ( $\mathrm{V}$ par craneal) y facial (VII par craneal) (Hamor y col 2000). El nervio trigémino proporciona sensibilidad a la glándula lagrimal (en concreto, a través del nervio lagrimal), las estructuras perioculares y el globo ocular. La estimulación de las terminaciones nerviosas de estas ramas del nervio trigémino trae como resultado la formación refleja de lágrimas controlada por fibras parasimpáticas derivadas del nervio facial (Nepp y col 2002).

Estudios realizados en humana demuestran (Eliason y col 2007) la eficacia de la técnica de electroacupuntura aplicada en acupuntos, entre ellos el Tai-yang, durante 15 minutos. En este estudio, el 88\% de 17 pacientes humanos tuvieron una mejoría estadísticamente significativa en los síntomas del ojo seco. Aunque diferimos de los autores en algunos aspectos de su trabajo, como la técnica de estimulación, coincidimos en que la aplicación de agujas sobre el acupunto Tai-yang produce un incremento en la secreción lagrimal.

Nuestro experimento en perros se asemeja a un estudio realizado en conejos (Gong y Sun 2007), utilizando los mismos acupuntos BL-2, Tai-yang y TH-23 donde fue demostrado que la estimulación de estos tres acupuntos puede aumentar significativamente la producción de lágrimas. En este trabajo, mediante estudio histológico de la glándula lagrimal, se demostró que el aumento de la producción de lágrimas, desde el momento de la aplicación y después de diez días la acupuntura, se debió a un incremento de la actividad secretora de la glándula.
Según nuestros resultados, realmente después de la estimulación de los acupuntos, se produce un incremento significativo de la secreción lagrimal medida con el TLS en el perro, pero no se puede indicar durante cuánto tiempo se mantienen elevados los niveles de lágrimas, ya que este estudio finaliza a los 60 minutos (T60) después de la retirada de las agujas. Sin embargo, en este tiempo (T60) se observa una disminución respecto del tiempo anterior (T30) por lo que se plantea una duda razonable de si ha comenzado un descenso de la secreción lagrimal hacia la normalización. Serían necesarios, por tanto, estudios donde las mediciones de TLS se realicen a más largo plazo.

En cualquier caso, ya que los tratamientos convencionales contra la hiposecreción lagrimal en el perro no son del todo satisfactorios y que los fármacos empleados para tratar la enfermedad deben ser aplicados durante semanas, meses e incluso de por vida para tener éxito, los resultados obtenidos por nosotros en el T30 abren una puerta a una nueva terapia única o en combinación con medicamentos que reduzca su frecuencia de aplicación.

Respecto de la PIO, distintos autores (Ulett y col 1998, Chu y Potter 2002, Kim y col 2005, Tangjitjaroen y col 2009) informan que cuando se aplica la técnica de acupuntura la reducción de la PIO se asocia con la disminución del humor acuoso debido a que los niveles de norepinefrina en la sangre son más bajos, lo que produce un descenso de la presión arterial, disminuye la actividad del sistema nervioso simpático y aumentan los niveles de endorfinas. El aumento de $\beta$-endorfinas en el humor acuoso después de la aplicación de las agujas 
sugiere que los opioides endógenos también podrían desempeñar un papel importante en la reducción de la inflamación a nivel ocular.

En nuestra experiencia, hemos observado que después de las técnicas de acupuntura los animales se sentían más relajados. Esto podría ser explicado por el incremento en los niveles de $\beta$-endorfinas y por la disminución de la presión arterial (Kurusu y col en 2005).

Experiencias realizadas por otros autores (Kim y col en 2005) demostraron con una estimulación de 20 minutos en perros sanos y con una única lectura a los 60 minutos, que la PIO se redujo en $2,2 \mathrm{mmHg}$, siendo estos resultados estadísticamente significativos. Aunque la técnica y los acupuntos utilizados en nuestro estudio son los mismos, diferimos de los autores respecto de que nosotros empleamos un tiempo de estimulación de 30 minutos. Pudimos observar cómo se produce una disminución progresiva de la PIO al realizar mayor cantidad de lecturas $(5,15$, 30 y $60 \mathrm{~min}$ ), alcanzando en el mismo tiempo T60 una disminución significativa de la PIO, con una reducción media de $8,05 \mathrm{mmHg}$, lo que nos lleva a pensar que el factor determinante en este caso pueda haber sido un mayor tiempo de estimulación.

En algún trabajo se ha utilizado estimulación de los acupuntos mediante electroacupuntura (Chu y Potter 2002), y se ha observado después de una hora de estimulación una reducción de la $\mathrm{PIO}$ de $9 \mathrm{mmHg}$, una disminución de la presión arterial sistémica y un descenso de la tasa de flujo del humor acuoso. Estas reducciones coincidieron con un aumento significativo de endorfinas en el humor acuoso, mientras que las concentraciones de los neurotransmisores simpáticos, la noradrenalina y la dopamina se redujeron. Según estos autores, el efecto de la hipotensión intraocular duró más de nueve horas. Sin embargo, con la técnica de acupuntura tradicional y los acupuntos utilizados en nuestro estudio, podemos comprobar que una hora después de la retirada de las agujas conseguimos una media de disminución de $8,05 \mathrm{mmHg}$, pero es cierto que no sabemos cuánto tiempo perduró esta disminución o cuándo regresó a sus valores basales.

Otros investigadores han realizado sus experiencias en pacientes con glaucoma clínico, o bien inducido experimentalmente. Así, se ha descrito que con la técnica de acupuntura se produjo una disminución de la PIO en perros a los que, previamente, se les había provocado glaucoma (Ralston 1977).

Estudios con humanos (Uhrig y col 2003) demostraron la reducción de la PIO en 18 pacientes con glaucoma e hipertensión ocular a los 15 minutos y 24 horas después de la estimulación con acupuntura, utilizando los mismos puntos que en nuestro trabajo ( $L I V-3, L I-4, G B-37)$. Sin embargo, otros autores (Meira-Freitas y col 2010) en un ensayo clínico controlado a doble ciego en humanos sanos, sobre el efecto de la acupuntura en la PIO, no encontraron ninguna variación significativa utilizando una estimulación de 20 minutos en los acupuntos $G B-1, G B-14$ y $B L-1$.
Comparando ambos trabajos con los resultados de nuestro estudio, estamos totalmente convencidos de que los puntos anatómicos para la colocación de las agujas son la clave para la obtención de buenos resultados terapéuticos. Sin embargo, los resultados del presente estudio no deben interpretarse como que la acupuntura puede curar o proteger los animales con presiones intraoculares altas que puedan inducir al glaucoma. Es necesario llevar a cabo más trabajos experimentales para dilucidar los mecanismos implicados en este tipo de terapia en relación con la liberación de sustancias como neuropéptidos y opioides endógenos tras la estimulación de los acupuntos.

Como conclusión de este trabajo experimental podemos decir que la técnica de acupuntura en el perro puede ser de gran utilidad en el tratamiento de procesos que cursen con sequedad ocular o aumento de la presión intraocular. Sin embargo, se deben realizar más ensayos clínicos en animales con patología ocular, aumentando el tiempo de las mediciones para verificar la duración de la respuesta y el tiempo de normalización de los efectos de las estimulaciones para apoyar estos resultados. Nosotros pensamos que la acupuntura, como tratamiento único o en combinación con otras terapias, puede ser una buena alternativa para disminuir los tiempos de tratamiento y favorecer sus resultados.

\section{REFERENCIAS}

Barton K, A Nava, DC Monroy, SC Pflugfelder. 1998. Cytokines and tear function in ocular surface disease. Adv Exp Med Biol 438, 461-469.

Bensky D, J O'Connor. 1981. Acupuncture: a comprehensive text. Eastland Press, Chicago, USA.

Burstein NL. 1985. The effects of topical drugs and preservatives on the tears and corneal epithelium in dry eye. Trans Ophthalmol Soc $U K$ 104, 402-409.

Cariello AJ, FH Casanova, AAS Lima, Y Juliano, A Tabosa. 2006. Effect of electroacupuncture to prevent selenite-induced cataract in Wistar rats. Arq Bras de Oftalmol 69, 299-303.

Cho SJ, O Kim. 2008. Acupuncture treatment for idiopathic Horner's syndrome in a dog. J Vet Sci 9, 117-119.

Chu TC, DE Potter. 2002. Ocular hypotension induced by electroacupuncture. J Ocul Pharmacol Th 18, 293-305.

Dabov S, G Goutoranov, R Ivanova, N Petkova. 1985. Clinical application of acupuncture in ophthalmology. Acupunct Electrhother Res 10, 79-93.

Eliason KJ, SC Richards, GT Schaumaun. 2007. Acupuncture treatment for dry eye. Medical Acupuncture 19, 25-28.

Ernest E. 2006. Acupuncture - a critical analysis. J Inter Med 259, 125-137.

Geerling G, S Maclennan, D Hartwig. 2004. Autologous serum eye drops for ocular surface disorders. Br J Ophthalmol 88, 1467-1474.

Gobbels M, M Spitznas. 1992. Corneal epithelial permeability of dry eyes before and after treatment with artificial tears. Ophthalmology 99, 873-878.

Gong L, X Sun. 2007. Treatment of intractable dry eyes: tear secretion increase and morphological changes of the lacrimal gland of rabbit after acupuncture. Acupunct Electrother Res 32, 223-233.

Grahn BH, ES Storey. 2004. Lacrimomimetics and lacrimostimulants. Vet Clin North Am Small Anim Pract 34, 739-753.

Gronlund MA, U Stenevi, T Lundeberg. 2004. Acupuncture treatment in patients with keratoconjunctivitis sicca: a pilot study. Acta Ophthalmol Scand 82, 283-290.

Hamor RE, SM Roberts, GA Severin, MJ Chavkin. 2000. Evaluation of results for Schirmer tear tests conducted with and without application 
of a topical anesthetic in clinically normal dogs of 5 breeds. Am J Vet Res 61, 1422-1425.

Kim MS, KM Seo, TC Nam. 2005. Effect of acupuncture on intraocular pressure in normal dogs. $J$ Vet Med Sci 67, 1281-1282.

Kim MS, JH Yoo, KM Seo, SM Jeong. 2007. Effects of electroacupuncture on intraocular pressure and hemodynamic parameters in isoflurane anesthetized dogs. J Vet Med Sci 69, 1163-1165.

Kurusu M, K Watanabe, T Nakazawa, T Seki, H Arai, H Sasaki, N Fuse, M Tamai. 2005. Acupuncture for patients with glaucoma. Explore (NY) 1, 372-376.

López-García JS, I García-Lozano, L Rivas, J Martínez-Garchitorena. 2007. Use of autologous serum in ophthalmic practice. Arch Soc Esp Oftalmol 82, 9-20.

Maggs D, P Millar, R Ofri. 2009. Glaucoma. En: Maggs D, Millar P, Ofri R (eds). Slatter -Fundamentos de Oftalmología Veterinaria. $4^{\mathrm{a}}$ ed. Elsevier Saunders, Barcelona, España.

Meira-Freitas D, AJ Cariello, RC Vita, A Tabosa, LA Melo. 2010. Short-term effect of acupuncture on intraocular pressure in healthy subjects. Acupunct Med 28, 25-27.

Müller LJ, CF Marfurt, F Kruse, TMT Tervo. 2003. Corneal nervios: estructura, contenido y función. Ojo Exp Res 76, 52-542.

Naruse S, K Mori, M Kurihara, N Nakajima, Y Matsumoto, S Kinoshita, Y Ohyama, T Nakamura. 2000. Chorioretinal blood flow changes following acupuncture between thumb and forefinger. Nihon Ganka Gakkai Zasshi 104, 717-723.

Nepp J, K Jandrasits, J Schauersberger, G Schild, A Wedrich, GL Sabine, A Spacek. 2002. Is acupuncture an useful tool for pain-treatment in ophthalmology? Acupunct Electrother Res 27, 171-182.
Ralston NS. 1977. Successful treatment and management of acute glaucoma using acupuncture. Am J Acupunct 5, 283-285.

Rodekohr S. 2002. Acupuntura y homeopatía en las pequeñas especies. Bases científicas y clásicas de terapéuticas complementarias. Asociación Mexicana de Médicos Veterinarios Especialistas en Pequeñas Especies 13, 128-134.

Ross J. 1989. Acupuncture. In: Zang FU (ed). The organ systems of traditional Chinese medicine. $2^{\text {nd }}$ ed. Churchill Livingstone, London, UK.

Tangjitjaroen W, J Shmalberg, PT Colahan, HS Xie. 2009. Equine acupuncture research: an update. J Equine Vet Sci 29, 698-709.

Tseng KL, HJ Liu, KY Tso, LC Woung, YC Su, JG Lin. 2006. A clinical study of acupuncture and SSP (silver spike point) electro-therapy for dry eye syndrome. Am J Chin Med 34, 197-206.

Uhrig S, J Hummelsberger, B Brinkhaus. 2003. Standardized acupuncture therapy in patients with ocular hypertension or glaucoma - results of a prospective observation study. Forsch Komplementarmed Klass Naturheilkd 10, 256-261.

Ulett GA, S Han, JS Han. 1998. Electroacupuncture: mechanisms and clinical application. Biol Psychiatry 44, 129-138.

Wilson SE, Q Liang, WJ Kim. 1999. Lacrimal gland HGF, KGF and EGF mRNA levels increase after corneal epithelial wounding. Invest Ophthalmol Vis Sci 40, 2185- 2190.

Xie H, V Preast. 2007. Xies Veterinary Acupuncture. Blackwell Publishing, Iowa, USA. 
